\title{
Recommender Framework for Sentimental Analysis using l-injection \\ ${ }^{1}$ P.Deepa, ${ }^{2}$ D.Eva Roshini, ${ }^{3}$ V.Dhivya Bharathi, ${ }^{4}$ S.Bhuvaneswari \\ ${ }^{1}$ Associate Professor, ${ }^{2,3,4}$ UG Scholars \\ Department of Computer Science and Engineering \\ Panimalar Engineering College, Chennai.
}

\begin{abstract}
We build up a novel structure, named as l-injection, to address the sparsity issue of recommender frameworks. Via precisely infusing low esteems to a chose set of unrated client thing sets in a client thing framework, we show that best $\mathrm{N}$ proposal correctness's of different community oriented separating (CF) systems can be altogether and reliably moved forward. We initially embrace the thought of pre-utilize inclinations of clients toward a tremendous measure of unrated things. Utilizing this thought, we distinguish uninteresting things that have not been evaluated yet but rather are probably going to get low appraisals from clients, and specifically ascribe them as low esteems. As our proposed approach is technique rationalist, it can be effectively connected to an assortment of $\mathrm{CF}$ calculations. Through extensive investigations with three genuine datasets (e.g., Movielens, Ciao, and Watcha), we show that our answer reliably and generally upgrades the exactnesses of existing CF calculations (e.g., thing based CF, SVDbased $\mathrm{CF}$, and $\mathrm{SVD++}$ ) by 2.5 to 5 times overall. Besides, our answer enhances the running time of those $\mathrm{CF}$ techniques by 1.2 to 2.3 times when its setting produces the best precision.
\end{abstract}

Keyword-Recommender systems, collaborative filtering, rating estimation methods, extensions to recommender systems.

\section{Introduction}

Huge information has developed as a broadly perceived pattern, drawing in considerations from government, industry and the scholarly world. As a rule, Big Data concerns substantial volume, mind boggling, developing informational collections with various, and self-ruling sources. Enormous Data applications where information accumulation has developed hugely and is past the capacity of generally utilized programming apparatuses to catch, oversee, and process inside a "mediocre slipped by time" is on the ascent. The most basic test for the Big Data applications is to investigate the vast volumes of information and concentrate valuable data or learning for future activities. Impelled by benefit registering and distributed computing, an expanding number of administrations are developing on the Internet. Therefore, benefit applicable information turn out to be too enormous to be successfully prepared by customary methodologies. In perspective of this test, a Clustering-based Collaborative Filtering approach (Club CF) is proposed in this paper, which goes for enrolling comparative administrations in similar bunches to suggest benefits cooperatively.

Group based proposal is best idea of as a variation on client based suggestion. Rather than prescribing things to clients, things are prescribed to bunch of comparative clients. This involves a pre-preparing stage, in which all clients are divided into bunches. Suggestions are then delivered for each bunch, to such an extent that the prescribed things are most intriguing to the biggest number of clients. The upside of this approach is that proposal is quick at runtime in light of the fact that practically everything is pre registered.

The most recent decade has seen a gigantic development of Web benefits as a noteworthy innovation for sharing information, figuring assets, and projects on the Web. With expanding reception and nearness of Web administrations, outlining novel methodologies for productive and successful Web benefit proposal has happened to 
principal significance. In existing web administrations revelation and proposal approaches concentrate on watchword overwhelming Web benefit web indexes, which have numerous impediments, for example, poor suggestion execution and substantial reliance on right and complex questions from clients. Late research endeavours on Web benefit suggestion fixate on two conspicuous methodologies: collective separating and substance based proposal. Lamentably, both methodologies have a few downsides, which confine their relevance in Web benefit suggestion. In proposed framework for suggestion we will utilize Agglomerative Hierarchal Clustering or Hierarchal Agglomerative Clustering for viable proposal in web-administrations. Our approach considers all the while both rating information (e.g., QoS) and semantic substance information (e.g., functionalities) of Web administrations utilizing a probabilistic generative model.

\section{Literature Survey}

1.Y.Koren in2009. Extensively, recommender frameworks depend on one of two techniques. The substance sifting approach makes a profile for every client or item to describe its inclination. For instance, a motion picture profile could incorporate traits with respect to its kind, the taking an interest performing artists, its film industry fame, et cetera. Client profiles may incorporate statistic data or answers gave on a reasonable poll. The profiles enable projects to connect clients with coordinating items. Obviously, content-based systems require gathering outer data that won't not be accessible or simple to gather on past client conduct-for instance, past exchanges or item evaluationswithout requiring the formation of express profiles. This approach is known as collective separating; a term instituted by the designers of Tapestry, the principal recommender system.1 Collaborative sifting breaks down connections amongst clients and interdependencies among items to recognize new client thing affiliations. The absolute most effective acknowledge of inert factor models depend on framework factorization. In its fundamental frame, lattice factorization describes the two things and clients by vectors of components deduced from thing rating designs. Such frameworks are especially valuable for amusement items, for example, films, music, and TV appears. Numerous clients will see a similar motion picture, and every client is probably going to see various diverse motion pictures. Clients have demonstrated willing to show their level of fulfillment with specific motion pictures, so a gigantic volume of information is accessible about which motion pictures request to which clients. Organizations can break down this information to prescribe motion pictures to specific clients.

2. S.Niwa in 2006. There have been numerous endeavors to develop page recommender frameworks utilizing communitarian sifting. In any case, the spaces these frameworks can cover are extremely confined on the grounds that it is exceptionally hard to gather client inclination information to site pages, and the quantity of site pages on the Internet is too vast. In this paper, we propose the best approach to develop another sort of page recommender framework covering everywhere throughout the Internet, by utilizing Folksonomy and Social Bookmark which are getting exceptionally prevalent in nowadays. We developed another web recommender framework which isn't constrained to specific sites, in view of vast measure of open bookmark information on SBM. We likewise use Folksonomy labels to arrange website pages and to express users 'preferences. By bunching Folksonomy labels, we can modify the reflection level of users 'preferences to the fitting level. We likewise tackled the issue of "tag repetition in Folksonomy "by grouping labels

3. R.Salakhutdinov in 2007. The vast majority of the current ways to deal with community oriented separating can't deal with substantial informational indexes. In this paper we demonstrate how a class of two-layer undirected graphical models, called Restricted Boltzmann Machines (RBM's), can be utilized to display forbidden information, for example, client's evaluations of motion pictures. We introduce proficient learning and induction methods for this class of models and show that RBM's can be effectively connected to the Netflix informational collection, containing more than 100 million client/film evaluations. We additionally demonstrate that RBM's marginally beat painstakingly tuned SVD models. At the point when the expectations of different RBM models and various SVD models are straightly consolidated, we accomplish a blunder rate that is well more than $6 \%$ superior to the score of Netflix's own framework. Low-rank approximations in view of limiting the aggregate squared separation can be discovered utilizing Singular Value Decomposition (SVD). In the synergistic separating space, in any case, a large 
portion of the informational indexes are scanty, and as appeared by Srebro and Jaakkola, this makes a troublesome non-raised issue, so a gullible arrangement isn't going work.1 In this paper we portray a class of two-layer undirected graphical models that sum up Restricted Boltzmann Machines to demonstrating unthinkable or tally information (Welling et al., 2005). Most extreme probability learning is unmanageable in these models; however we demonstrate that learning can be performed effectively by following estimation to the inclination of an alternate target work called "Contrastive Divergence"

4. Yu Zhang, Bin Cao, Dit-Yan Yeung in 2010. Community oriented separating is a compelling proposal approach in which the inclination of a client on a thing is anticipated in light of the inclinations of different clients with comparative interests. A major test in utilizing communitarian separating techniques is the information sparsity issue which regularly emerges in light of the fact that every client ordinarily just rates not very many things and subsequently the rating framework is to a great degree meager. The MCF issue is especially appropriate for huge scale web based business and interpersonal interaction administrations which frequently give a differing scope of items or administrations. CF methods abuse past exercises of the clients, for example, their exchange history or item fulfillment communicated in appraisals, to anticipate the future exercises of the clients. CF-based proposal frameworks have turned out to be progressively prevalent in light of the fact that it is for the most part significantly less demanding to gather the past exercises of clients than their profiles, halfway because of protection contemplations. The MCF issue is especially reasonable for vast scale online business and informal communication administrations which frequently give a differing scope of items or administrations. The adequacy of abusing the connections between various areas. A multi-space CF (MCF) issue which mutually models an accumulation of rating expectation undertakings emerging from different areas.

5. L. Jeba Sheela in 2016 twitter is an online social networking site which contains rich amount of data that can be a structured, semi-structured and un-structured data. In this work, a method which performs classification of tweet sentiment in Twitter is discussed. To improve its scalability and efficiency, it is proposed to implement the work on Hadoop Ecosystem, a widely-adopted distributed processing platform using the Map Reduce parallel processing paradigm. Finally, extensive experiments will be conducted on realworld data sets, with an expectation to achieve comparable or greater accuracy than the proposed techniques in literature.

\section{III.System Design}

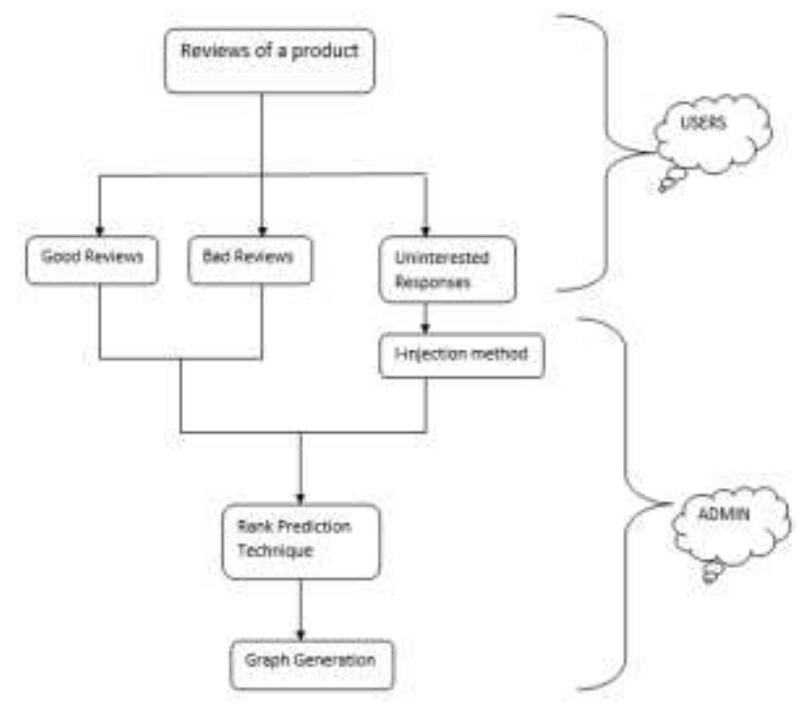

Figure 1Architecture Diagram

The customer views the product and if he/she wishes it gets it, they view the reviews. There are both good and bad reviews and it also shows the products that doesn't have any rating it is called the uninterested products. The uninterested products are distinguished from others by using 1injection method, which is done by the admin. All the reviews are been joined and are given rating by using the Rank Prediction technique. On the whole, finally a graph is generated based on the ratings given by the customers.

\section{Proposed System}

Collaborative Filter Algorithm (utilized for separating the uninterested things) the principle distinction between collective sifting and substance based separating is theoretical. Where content-based separating is worked around the traits of a given question, shared sifting depends on the conduct of clients. This approach has some particular focal points over substance based sifting:

It profits by expansive client bases. Basically, the more individuals are utilizing the administration, the better your suggestions will progress toward becoming, without doing extra advancement work or depending on branch of knowledge mastery. 
It's adaptable crosswise over various areas. Cooperative sifting approaches are appropriate to exceedingly assorted arrangements of things. Where content-construct channels depend in light of metadata, community oriented sifting depends on genuine action, enabling it to make associations between apparently divergent things (like say, a detachable engine and an angling pole bar) that in any case may be applicable to some arrangement of clients (for this situation, individuals who jump at the chance to angle).

It delivers more fortunate suggestions. With regards to suggestions, exactness isn't generally the most astounding need. Content-based separating approaches tend to indicate clients things that are fundamentally the same as things they've effectively enjoyed, which can prompt channel bubble issues. By differentiate, most clients have interests that traverse distinctive subsets, which in principle can bring about more assorted (and fascinating) proposals.

It can catch more subtleties around things. Indeed, even a profoundly definite substance based separating framework will just catch a portion of the highlights of a given thing. By depending on real human experience, collective sifting can now and again prescribe things that have a more prominent partiality with each other than a strict correlation of their traits would recommend.

Rank Prediction Technique (used to demonstrate the best rank products) the attributes of various kinds of web crawlers physically are characterized sets of watchwords. We compressed the measurements of each gathering watchwords. The means of taking in the inquiries: 1) word division; 2) de-noising and removing the spine catchphrases. In the event that some question words seem at least one gathering of catchphrases in meaning of guidelines, showing these sorts of web index comes about than alternate kinds will probably require clients required. Subsequently, it is a scoring component to impact the general score in procedure of accumulation of different sorts of results.

\section{MODULE DESCRIPTION}

\section{A. Admin Login}

This module utilized for login strategy like will you enter administrator name and secret word is right means enter inside site. If not right client name or watchword then "invalid client name or secret key this message will come".

\section{B. File Uploads}

This module administrator transfers all items. Here those fields enter like item name, mark, date, amount, picture, and cost. Administrator can transfer datasets too.

\section{View All Users}

Administrator can see every one of clients' subtle elements. These clients all points of interest originate from client enrollment.

\section{View All Products}

Administrator Added all items administrator can see this module. Here view that items rank and amount likewise see here. What's more, administrator don't need that item erase likewise there.

\section{E. Positive Polarity}

These modules have positive extremity. Positive extremity implies client will give any survey positive means see here. In the event that client will give that audit negative means see another module of negative module

Here one grouping utilized for item based survey, that arrangement like substance based channel the all kind of sentence than after that audit split the word by word and will checking these word positive word or negative word if positive means see here.

\section{F. Negative Polarity}

These modules have negative extremity. Negative extremity implies client will give any survey negative means see here. Here additionally characterization utilized for item based audit, that grouping like channel the all kind of sentence these sentence originate from client bought item based given that surveys. Than after that audit split the word by word and will checking these word positive word or negative word if negative means see here.

\section{G. Over all Result for Products}

Administrator can see all items name, positive positioning tally and negative checks like numeric's. On the off chance that negative esteems high and positive esteem is low that items over all outcomes is Bad Products. In the event that positive esteems high negative esteems is low that items result is Good Products. On the off 
chance that Positive and Negative is equivalent is that item result is Neutral Products.

\section{H. Graph}

Administrator see diagram originate from general outcome points of interest. Here diagram in light of the intrigued items and $\mathrm{UN}$ intrigued items positioning based view here.

\section{Interested Products}

The most clients bought or like best level items considered for clients intrigued items. At that point these intrigued items have a positions, subtle elements, and pictures.

\section{J. Uninterested Products}

The all client's UN like items considered for client's UN intrigued items. At that point these intrigued items have a positions, subtle elements, and pictures.

\section{K. Users}

This Module utilized for new enlistment. Here used to username, watchword, versatile, email, and address this field. On the off chance that you are not enlisted here you can't enter this application. At that point clients enrolled that time his get client name and proprietor key from enlisted account subtle elements in his mail.

\section{Login}

This module utilized for login method like will you enter username and secret key is right means enter inside site. If not right client name or secret word then "invalid client name or watchword this message will come".

M. View all products and Recommendations

This module uses of admin uploaded all product view here. If users want purchase any products. And users can view products recommendation also, this recommendation products view based on the above $80 \%$ rating or raking products only.

\section{N. Review}

Here users after purchase products then only users can give review for those products.

\section{IMPLEMENTATION}

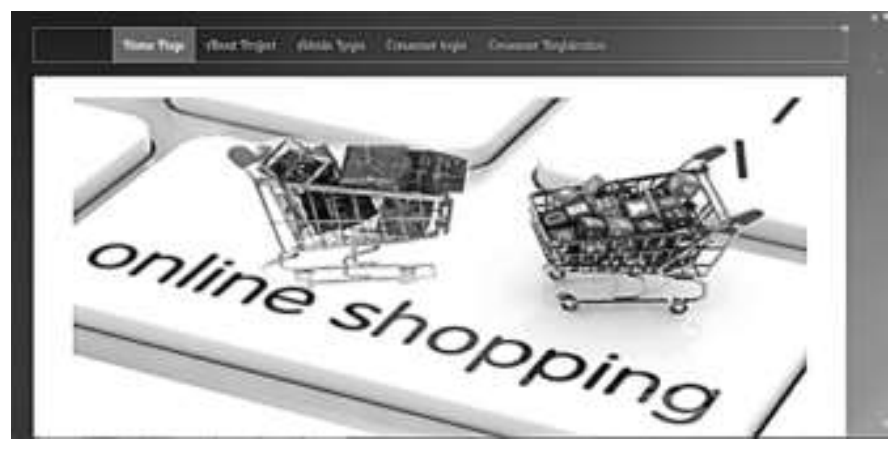

Figure 2 home page

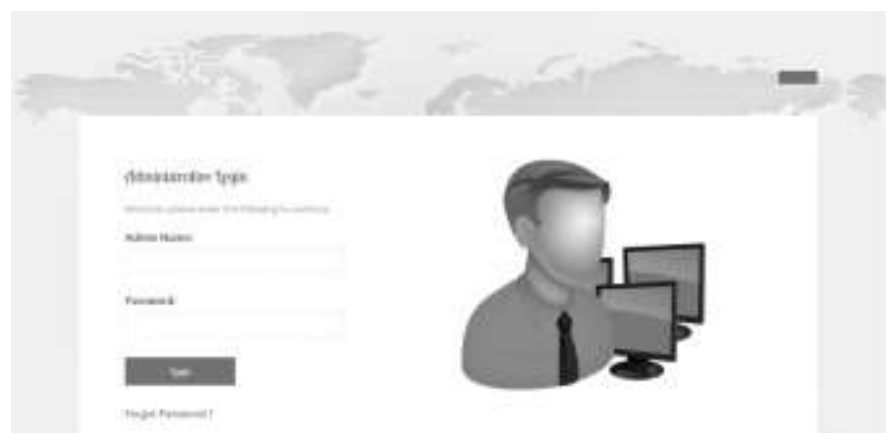

Figure 3 Admin home

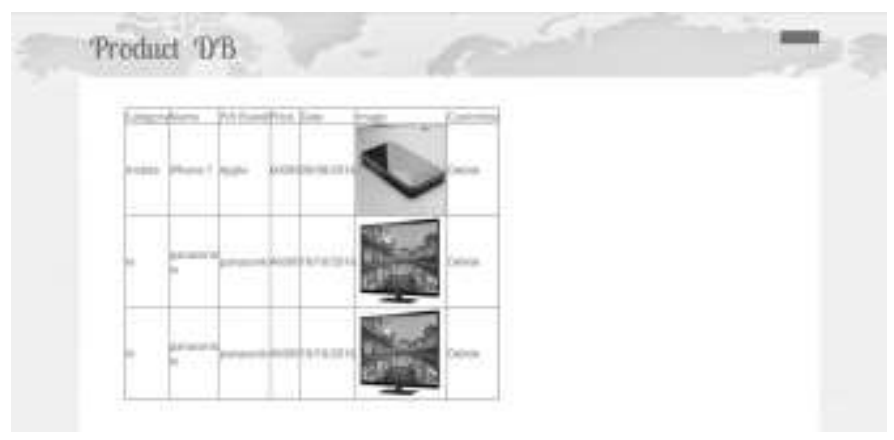

Figure 4 View product page

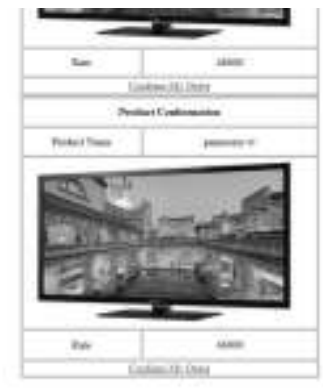

Figure 5 View product detail

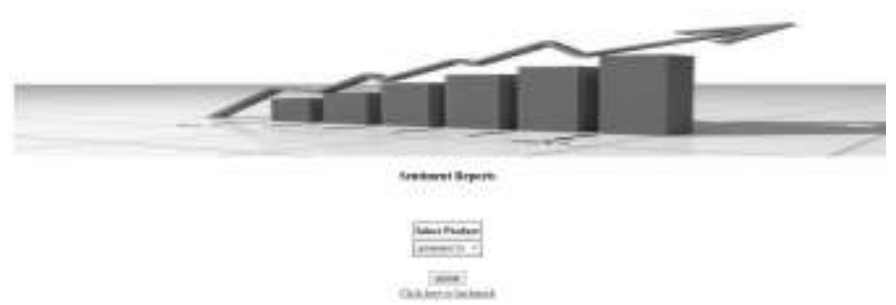


Figure 6 Sentiment report

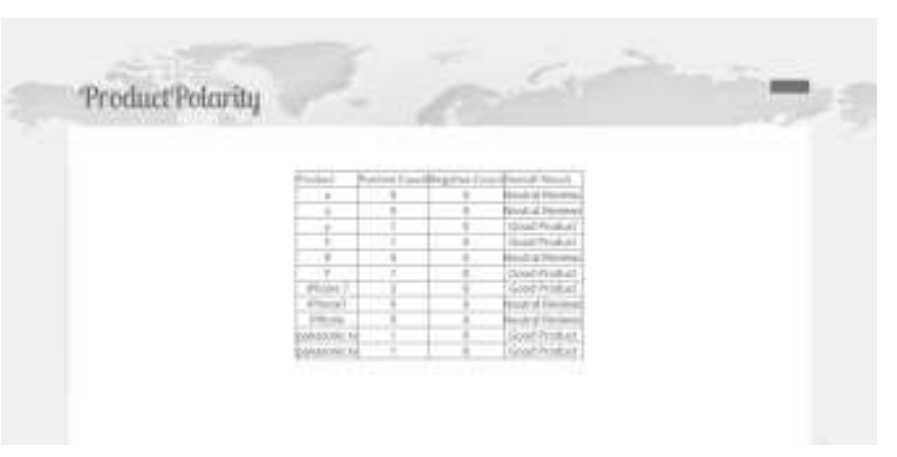

Figure 7 Sentiment analysis

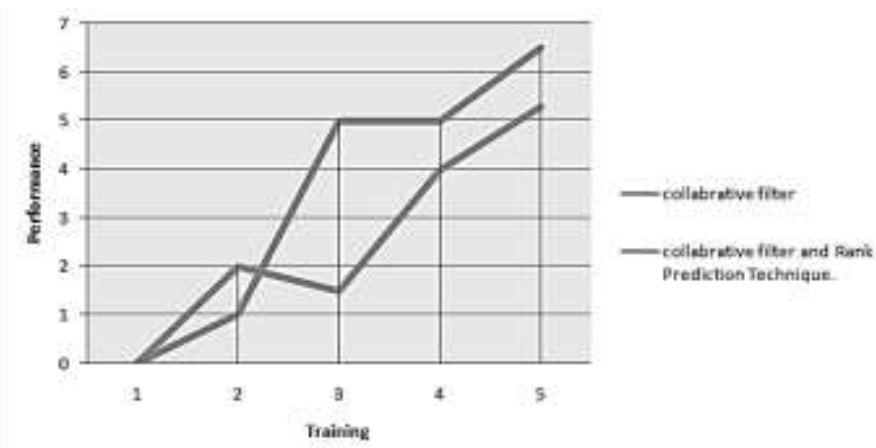

Figure 8 Performance Analysis Report

\section{VII.Conclusion}

We display a Club $\mathrm{CF}$ approach for huge information applications significant to benefit proposal. Before applying CF system, administrations are converted into a few groups by means of an AHC calculation. At that point the rating likenesses between administrations inside a similar group are registered. As the quantity of administrations in a bunch is substantially less than that of in the entire framework, Club CF costs less online calculation time. In addition, as the appraisals of administrations in a similar group are more applicable with each other than with the ones in different bunches. On the off chance that we need, include the new motion pictures with name, classification and year

\section{Future Work}

We improve the efficiency of successfully demonstrated that the proposed approach is effective and practical, dramatically improving the accuracies of existing CF methods by 2.5 to 5 times.

\section{Reference}

[1] Y. Koren et al., "Matrix factorization techniques for recommender systems," IEEE Computer, vol. 42, no. 8, pp. 30-37, 2009.
[2] V.Sindhwani et al., "A family of nonnegative matrix factorization for one-class collaborative filtering," in Proc. of ACM RecSys, 2009.

[3] L.Jeba Sheela., "A Review of Sentiment Analysis in Twitter Data Using Hadoop," International Journal of Database Theory and Application Vol.9, No.1 2016, pp.7786.

[4] J. Tang, H. Gao, and H. Liu, "mtrust: discerning multi-faceted trust in a connected world," in Proc. of WSDM", pages $=93-102$, year $=2012$.

[5] J. Lee, D. Lee, Y. Lee, W. Hwang, and S. Kim, "Improving the

[6] accuracy of top-n recommendation using a preference model," Inf. Sci., vol. 348, pp. 290-304, 2016.

[7] R.Salakhutdinov et al., "Restricted boltzmann machines for collaborativefiltering," in Proc. of ACM ICML, 2007, pp. 791-798.

[8] R. Salakhutdinov and A. Mnih, "Probabilistic matrix factorization," in Proc. of NIPS, 2007, pp. 1257-1264.

[9] V.Sindhwani, S. S. Bucak, J. Hu, and A. Mojsilovic, "One-class matrix completion with low-density factorizations," in Proc. IEEE ICDM, Dec. 2010, pp. 1055-1060.

[10] M.Jiang, P. Cui, X. Chen, F. Wang, W. $\mathrm{Zhu}$, and S. Yang, "Social recommendation with cross-domain transferable knowledge," IEEE Trans. Knowl. Data Eng., vol. 27, no. 11, pp. 3084-3097, Nov. 2015.

[11] Y Koren, "Factorization meets the neighborhood: A multifaceted collaborative filtering model," in Proc. ACM KDD, 2008, pp. 426-434.

[12] Y. Zhang, B. Cao, and D.-Y. Yeung, "Multi-domain collaborative filtering," in Proc. 26th Conf. Uncertainty Artif. Intell. (UAI), Catalina Island, CA, USA, 2010, pp. 725-732.

[13] H.Steck, "Training and testing of recommender systems on data missing not at random," in Proc. ACM KDD, 2010, pp. 713-722.

[14] X. Yang, H. Steck, Y. Guo, and Y. Liu, "On top-k recommendation using social networks," in Proc. ACM RecSys, 2012, pp. 67-74. 